\title{
CONVERSE SAMPLING AND INTERPOLATION
}

\author{
J. R. HIGGINS \\ I.H.P. \\ 4 RUE DU BARY \\ 11250 MONTCLAR \\ FRANCE
}

\begin{abstract}
The present note focuses on ideas of converse sampling and related ideas of interpolation. The presentation is in the setting of reproducing kernel Hilbert space. Sources are found in the classical literature, going back to 1920.
\end{abstract}

Key words and phrases : sampling, converse sampling, interpolation, reproducing kernel Hilbert space.

2010 AMS Mathematics Subject Classification - 94A20, 41A05, 46E22.

\section{INTRODUCTION}

Converse sampling and interpolation are considered in the setting of reproducing kernel Hilbert space (we shall write RKHS).

Theorem 1 recalls a direct sampling theorem in RKHS; Theorem 2 has both converse sampling and interpolatory components; Theorem 3 concerns the uniqueness of a band-limited interpolant to given data, when it exists; Theorem 4 gives a 'minimum norm' condition for the existence and uniqueness of an interpolant to given data.

The classical case is tracked through the discussion.

Sources are found in the classical works of $\operatorname{Ogura}^{1}[10,11]$ dating from 1920, J.M. Whittaker [14, 15] from 1929, Kotel'nikov [9] from 1933, Yen [17] from 1956 and Yau [16] from 1967. Thus the ideas discussed here have roots in the distant past.

We follow the RK theory as developed by Saitoh in, e.g., [13]. See [12] and many references found there for effectively the same theory of RK s in the setting of time series analysis. See also [4].

\footnotetext{
${ }^{1}$ Ogura was in the habit of writing a dateline at the end of his papers, from which it appears that in March 1919 he had completed the paper [10], which included a form of the classical direct sampling theorem and an indication of how to prove it by residue calculus. Later, in July 1919, he had completed [11] which included a converse theorem [11, p. 240]. Actually, Ogura presented it as being converse to a theorem of E.T. Whittaker but there is some obscurity surrounding this (see [5]).
} 
Other settings in which converse sampling is treated are [3] (locally compact abelian groups), [2] (second-order stationary stochastic processes) and [6] (the Kramer sampling theory).

\section{Preliminaries and Notations}

Let us assume sufficient background for the following definitions and assertions to hold. The reader is referred to [13] for details.

First, $\theta$ will always denote the null element of a space. Let $E$ be an arbitrary set; let $\mathfrak{H}$ be a separable Hilbert space and let $\kappa_{t}$ be a mapping of $E$ into $\mathfrak{H}$. Let $\kappa_{t}$ generate a kernel $k$ on $E \times E$ by $k(s, t):=\left\langle\kappa_{t}, \kappa_{s}\right\rangle_{\mathfrak{H}}$. Then the image $R_{k}$ of $\mathfrak{H}$ under the linear transformation $\mathcal{L}$ defined by $(\mathcal{L} \rho)(t):=\left\langle\rho, \kappa_{t}\right\rangle_{\mathfrak{H}}$ is an RKHS with reproducing kernel $k$. For any $f \in R_{k}$ there will be one and only one $\varphi$ such that $f=\mathcal{L} \varphi$. Members of $R_{k}$ can be called band-limited in the $R K$ sense, extending the classical usage (see Example - the classical case (below)).

Concerning summation notations, let

$$
\sum_{n \in \mathbb{Z}} \alpha_{n}:=\lim _{N \rightarrow \infty} \sum_{|n| \leq N} \alpha_{n} .
$$

Now $\mathbb{X} \subset \mathbb{Z}$ will always denote an indexing set, and

$$
\sum_{n \in \mathbb{X}} \alpha_{n}:=\lim _{N \rightarrow \infty} \sum_{N} \alpha_{n}:=\lim _{N \rightarrow \infty} \sum_{|n| \leq N, n \in \mathbb{X}} \alpha_{n} .
$$

The notation $\sum_{n \in \mathbb{Z}}^{\prime} \alpha_{n}$ indicates that $\alpha_{0}=0$.

Let $\left\{\left(t_{n}, c_{n}\right)\right\},(n \in \mathbb{X})$, be given, where $\left(t_{n}, c_{n}\right) \in E \times \mathbb{C}$. We call

$$
\sum_{n \in \mathbb{X}} c_{n} k\left(t, t_{n}\right)
$$

a cardinal series (following J.M. Whittaker). If $f: E \rightarrow \mathbb{C}$ be given, then in particular we call

$$
\sum_{n \in \mathbb{X}} f\left(t_{n}\right) d_{n} k\left(t, t_{n}\right)
$$

a sampling series for $f$. Here $\left\{d_{n}\right\}$ may, for example, arise from normalizing factors, as e.g. in (5).

Let $T:=\left\{t_{n}\right\} \subset E,(n \in \mathbb{X})$, denote a set of distinct points of $E$. When it is supposed that $T$ is such that $\left\{\kappa_{t_{n}}\right\}$ is an orthogonal set in $\mathfrak{H}$ we can hardly expect it to come already normalized, since $\kappa_{t}$ must be defined before the assumption concerning $T$ is made. Consequently, normalising factors $\mu_{n}=\left\|\kappa_{t_{n}}\right\|_{\mathfrak{H}}^{-1}$ must be introduced so that $\left\{\mu_{n} \kappa_{t_{n}}\right\}$ is an orthonormal set.

Let $\left\{\mu_{n} \kappa_{t_{n}}\right\}$ be an orthonormal basis for $\mathfrak{H}$ and let (1) be convergent. Then the cardinal series (1) is a sampling series for its sum. We denote this sum by 
$F$. Since (1) converges in norm it converges pointwise over $E$ by the standard principle in RKHS [13, p. 36, Theorem 3]. Now from (1),

$$
F\left(t_{m}\right)=\sum_{n \in \mathbb{X}} c_{n} k\left(t_{m}, t_{n}\right)=\sum_{n \in \mathbb{X}} c_{n}\left\langle\kappa_{t_{n}}, \kappa_{t_{m}}\right\rangle=\sum_{n \in \mathbb{X}} c_{n}\left(\mu_{m} \mu_{n}\right)^{-1} \delta_{n m}=c_{m} \mu_{m}^{-2} .
$$

Then (1) becomes a sampling series of the form (2).

Then $F$ is an interpolant to the data $\left\{\left(t_{n}, c_{n}\right)\right\}$, up to normalising factors. If it can be shown that an interpolant belongs to $R_{k}$ then it can be called a band-limited interpolant.

Any circumstances which would allow the representation of $f$ by a sampling series to imply that $f \in R_{k}$ would have the nature of a converse to Theorem 1 (below).

The presence of a converse to the classical sampling theorem was recognized by Kotel'nikov [9]: if $f$ is represented by the sampling series (7) then it is band-limited. His attractive argument is, effectively, that since each term of the sampling series (7) is band-limited, then its sum must also be band-limited.

However, these ideas of sampling, converse sampling and interpolation do not seem to admit any very precise classification.

Remark 1. We need only impose the assumption that there exists a set of points $T$ such that $\left\{\kappa_{t_{n}}\right\}$ is complete in $\mathfrak{H}$ to obtain a set of uniqueness for $R_{k}$, that is, $f\left(t_{n}\right)=0$ for every $n$ implies that $f=\theta$; and conversely. Indeed, let $f \in R_{k}$ so that there is one and only one $\varphi \in \mathfrak{H}$ such that

$$
f(s)=\left\langle\varphi, \kappa_{s}\right\rangle_{\mathfrak{H}}, \quad(s \in E) .
$$

Let $f\left(t_{n}\right)=\left\langle\varphi, \kappa_{t_{n}}\right\rangle_{\mathfrak{H}}=0$ for every $n$. Then by the completeness of $\left\{\kappa_{t_{n}}\right\}, \varphi$ is null, hence from (4), $f$ is null.

The converse is even simpler. Let $T$ be a set of uniqueness for $R_{k}$. Then

$$
f\left(t_{n}\right)=\left\langle\varphi, \kappa_{t_{n}}\right\rangle_{\mathfrak{H}}=0
$$

for every $n$ implies that $f$ is null, and hence that $\varphi$ is null. But this is the condition that $\left\{\kappa_{t_{n}}\right\}$ be complete.

See [7, p. 76] or [13, p. 189] for the following:

Theorem 1 (Direct sampling theorem). Let $\mathbb{X}$ be an indexing set, and let $T$ be such that $\left\{\varphi_{n}\right\}:=\left\{\mu_{n} \kappa_{t_{n}}\right\}$ is an orthonormal basis for $\mathfrak{H}$. Then

(i) $T$ is a set of uniqueness for $R_{k}$;

(ii) for every $f \in R_{k}$ and $t \in E$,

$$
f(t)=\sum_{n \in \mathbb{X}} f\left(t_{n}\right) \mu_{n}^{2} k\left(t, t_{n}\right),
$$

converging in the norm of $R_{k}$ and pointwise over $E$.

Example-the classical case. The classical sampling theorem is, of course, a special case of this; one takes $E=\mathbb{R} ; t_{n}=n,(n \in \mathbb{Z}) ; \mathfrak{H}=L^{2}(-\pi, \pi)$; 
$\kappa_{t}=e^{-i t(\cdot)} ; \mu_{n}=(2 \pi)^{-1 / 2},(n \in \mathbb{Z})$ and $\mathcal{L}$ to be the (inverse) $L^{2}$ Fourier transform $\mathcal{F}^{-1}$. Then $R_{k}$ is the classical Paley-Wiener space

$$
P W:=\left\{f: f \in L^{2}(\mathbb{R}) \cap C(\mathbb{R}) ;(\mathcal{F} f)(\omega)=0 \text {, a.a. } \omega \notin[-\pi, \pi]\right\}
$$

and $\mathbb{Z}$ is a set of uniqueness for $P W$. In particular an $f$ satisfying the Fourier transform condition in (6) is called band-limited (in the classical sense).

The reproducing kernel is $2 \pi \sin \pi(t-s) / \pi(t-s)$, and (5) becomes the classical sampling series for $f \in P W$ :

$$
f(t)=\sum_{n \in \mathbb{Z}} f(n) \frac{\sin \pi(t-n)}{\pi(t-n)}
$$

converging in the norm of $L^{2}(\mathbb{R})$ and pointwise over $\mathbb{R}$ in the sense of the limit of symmetric partial sums.

\section{A MiXed CONVERSE AND INTERPOLATION RESUlT}

With the notations already established we have

Theorem 2. Let $\left(x_{n}\right) \in \ell^{2},(n \in \mathbb{X})$, where $\left(x_{n}\right) \neq \theta$. Let $T:=\left\{t_{n}\right\} \subset E$ be such that $\left\{\kappa_{t_{n}}\right\}$ (at least one of which is not null) is an orthogonal subset of $\mathfrak{H}$ so that $\left\{\mu_{n} \kappa_{t_{n}}\right\}$ is orthonormal. Then the cardinal series

$$
\sum_{n \in \mathbb{X}} x_{n} \mu_{n} k\left(t, t_{n}\right)
$$

converges in the norm of $R_{k}$, and pointwise over $E$, to a sum $f$ of the form

$$
f(t)=(\mathcal{L} \varphi)(t)=\left\langle\varphi, \kappa_{t}\right\rangle_{\mathfrak{H}}, \quad(t \in E),
$$

where

$$
\begin{aligned}
\varphi & :=\sum_{n \in \mathbb{X}} x_{n} \mu_{n} \kappa_{t_{n}} . \\
f\left(t_{m}\right) & =\frac{x_{m}}{\mu_{m}}, \quad(m \in \mathbb{X}) .
\end{aligned}
$$

(iii) If, in addition, $T$ is a set of uniqueness for $R_{k}$, then $\left\{\kappa_{t_{n}}\right\}$ is complete and hence a basis for $\mathfrak{H}$.

Proof. It follows at once from the Riesz-Fischer theorem that the series (10) converges in $\mathfrak{H}$. To prove part (i), we show that (8) converges in $R_{k}$ to the sum $f(t)$ given in (9), where $\varphi$ is given by (10). Indeed, for $N>0$,

$$
\begin{aligned}
& \left\|\left\langle\varphi, \kappa_{(\cdot)}\right\rangle_{\mathfrak{H}}-\sum_{N} x_{n}\left(\mathcal{L} \mu_{n} \kappa_{t_{n}}\right)(\cdot)\right\|_{R_{k}}=\left\|(\mathcal{L} \varphi)(\cdot)-\sum_{N} x_{n} \mu_{n}\left(\mathcal{L} \kappa_{t_{n}}\right)(\cdot)\right\|_{R_{k}} \\
= & \left\|\left(\mathcal{L}\left\{\varphi-\sum_{N} x_{n} \mu_{n} \kappa_{t_{n}}\right\}\right)(\cdot)\right\|_{R_{k}}=\left\|\varphi-\sum_{N} x_{n} \mu_{n} \kappa_{t_{n}}\right\|_{\mathfrak{H}} \rightarrow 0 \text { as } N \rightarrow \infty .
\end{aligned}
$$

Pointwise convergence holds by the standard principle in RKHS. 
To prove part (ii) we find that, since (8) converges pointwise over $E$, we have for every $m$,

$$
f\left(t_{m}\right)=\sum_{n \in \mathbb{X}} x_{n}\left(\mathcal{L} \mu_{n} \kappa_{t_{n}}\right)\left(t_{m}\right)=\sum_{n \in \mathbb{X}} x_{n}\left\langle\mu_{n} \kappa_{t_{n}}, \kappa_{t_{m}}\right\rangle_{\mathfrak{H}}=\sum_{n \in \mathbb{X}} \frac{x_{n}}{\mu_{m}} \delta_{m, n}=\frac{x_{m}}{\mu_{m}} .
$$

Part (iii) follows from the last part of Remark 1.

\section{Remarks on Theorem 2.}

Had the condition $\left(x_{n}\right) \in \ell^{p}, 1 \leq p \leq 2$, been assumed in Theorem 2 instead of $\left(x_{n}\right) \in \ell^{2}$, the same result would have been obtained since $\ell^{p} \subset \ell^{2}$. In fact, Ogura [11, Theorem III] assumed the $\ell^{1}$ condition.

Theorem 2 has the nature of both a converse sampling and an interpolation theorem. Component (i) is the converse part since the convergence of (8) implies that its sum is band-limited. Component (ii) is the interpolatory part since it shows that the sum in (8) is an interpolant.

Example. This is a continuation of the Classical Example in $\S 2$, and we continue with the notations adopted there. We recall that $\left\{\mu_{n} \kappa_{t_{n}}\right\}=\left\{\frac{1}{\sqrt{2 \pi}} e^{-i n(\cdot)}\right\}$ is an orthonormal basis for $L^{2}(-\pi, \pi)$, and assume that $\left(x_{n}\right) \in \ell^{2}$. Then

(i) equation (8) becomes

$$
\sum_{n \in \mathbb{Z}} x_{n} \frac{1}{\sqrt{2 \pi}} 2 \pi \frac{\sin \pi(t-n)}{\pi(t-n)}=\sqrt{2 \pi} \sum_{n \in \mathbb{Z}} x_{n} \frac{\sin \pi(t-n)}{\pi(t-n)},
$$

converging in the norm of $L^{2}(\mathbb{R})$, and pointwise over $\mathbb{R}$, to a function $f$ of the form

$$
f(t)=\int_{-\pi}^{\pi} \varphi(\gamma) e^{i t \gamma} d \gamma
$$

where

$$
\begin{aligned}
& \varphi(\gamma)=\frac{1}{\sqrt{2 \pi}} \sum_{n \in \mathbb{Z}} x_{n} e^{-i n \gamma} . \\
& f(m)=\sqrt{2 \pi} x_{m}, \quad(m \in \mathbb{Z}) .
\end{aligned}
$$

Theorem 2 shows that the function $f$ in (12) is a band-limited interpolant. In the classical case $f$ can sometimes be calculated explicitly in terms of a data sequence $\left(x_{m}\right)$. For example, suppose that for some $h \in \mathbb{N}, x_{n}=(i n)^{-h}$, $(n \in \mathbb{Z} \backslash\{0\})$. Then the series (13) becomes

$$
\sum_{n \in \mathbb{Z}}^{\prime} \frac{e^{-i n \gamma}}{(i n)^{h}}, \quad h=1,2, \ldots,
$$

whose sum for any particular $h$ can be calculated directly as being piecewise polynomial of degree $h$ on $(-\pi, \pi)$. Details for this class of examples can be found in $[18$, p. 17], 


\section{UNIQUENESS OF A BAND-LIMITED INTERPOLANT}

With the notations already established we have

Theorem 3. Let $T \subset E$ be such that $\left\{\mu_{n} \kappa_{t_{n}}\right\},(n \in \mathbb{X})$, is an orthonormal basis for $\mathfrak{H}$. Let $\left(z_{n}\right)$ be given. There does not necessarily exist an $F \in R_{k}$ that interpolates these data, i.e., $F\left(t_{n}\right)=z_{n} / \mu_{n}$ for every $n$. But if such an interpolant $F$ does exist, it is unique and $\left(z_{n}\right) \in \ell^{2}$.

Proof. If such an $F$ exists in $R_{k}$, we can expand $F$ by the direct sampling theorem (Theorem 1):

$$
F(t)=\sum_{n \in \mathbb{X}} F\left(t_{n}\right) \mu_{n}^{2} k\left(t, t_{n}\right)=\sum_{n \in \mathbb{X}} z_{n} \mu_{n} k\left(t, t_{n}\right) .
$$

We find that $\left(z_{n}\right)$ are the coefficients in an orthonormal expansion, therefore Bessel's inequality applies and $\left(z_{n}\right) \in \ell^{2}$.

As to the uniqueness, suppose that there exists a $G \in R_{k}$ for which $G\left(t_{n}\right)=$ $F\left(t_{n}\right),(n \in \mathbb{X})$. Since $G \in R_{k}$, there exists a $\psi \in \mathfrak{H}$ such that $G=\mathcal{L} \psi$. Then for every $n \in \mathbb{X}$,

$$
\begin{gathered}
0=G\left(t_{n}\right)-F\left(t_{n}\right)=(\mathcal{L} \psi)\left(t_{n}\right)-(\mathcal{L} \varphi)\left(t_{n}\right) \\
=\left\langle\psi, \kappa_{t_{n}}\right\rangle_{\mathfrak{H}}-\left\langle\varphi, \kappa_{t_{n}}\right\rangle_{\mathfrak{H}}=\left\langle\psi-\varphi, \kappa_{t_{n}}\right\rangle_{\mathfrak{H}} .
\end{gathered}
$$

Hence $\psi=\varphi$ by the completeness of the basis $\left\{\kappa_{t_{n}}\right\}$, therefore $G \equiv F$.

\section{A 'MINIMUM NORM' CONDITION}

When $\left(x_{n}\right) \in \ell^{2}$ we saw in $\S 3$ that, under the hypotheses of Theorem 2 , the cardinal series (8) converges to a unique band limited interpolant. When $\left(x_{n}\right)$ is given we saw in $\S 4$ that, under the hypotheses of Theorem 3 , the uniqueness of a band limited interpolant is consequent upon its existence.

To round off this train of thought, suppose we assume only that $H$ is a RKHS of continuous complex valued functions on a set $E$; and that two data sequences be given: $\left(x_{n}\right), x_{n} \in \mathbb{C}$ and $\left(t_{n}\right), t_{n} \in E,(n \in \mathbb{X})$. In Theorem 4 below we find that a 'minimum norm' condition singles out a unique interpolant.

Theorem 4. Let I be the class of all interpolants, i.e.,

$$
I:=\left\{f \in H: f\left(t_{m}\right)=x_{m},(m \in \mathbb{X}) .\right\}
$$

If I is not empty it contains a member of smallest norm, which is unique.

Proof. I is closed. In fact we show that it is sequentially closed. Let $\left\{g_{n}\right\} \subset I$, $(n \in \mathbb{N})$, converge to $g \in H$. Then $\left\{g_{n}\right\}$ converges pointwise over $E$ to $g$ by the standard principle. Hence,

$$
\left|x_{m}-g\left(t_{m}\right)\right|=\left|g_{n}\left(t_{m}\right)-g\left(t_{m}\right)\right| \rightarrow 0 \text { as } n \rightarrow \infty, \quad(m \in \mathbb{X}) .
$$

Therefore $x_{m}=g\left(t_{m}\right),(m \in \mathbb{X})$, so that $g \in I$. 
$I$ is convex. Let $c_{1}, c_{2} \geq 0$, let $c_{1}+c_{2}=1$ and let $f_{1}, f_{2} \in I$. Then for every $m \in \mathbb{X}$,

$$
c_{1} f_{1}\left(t_{m}\right)+c_{2} f_{2}\left(t_{m}\right)=\left(c_{1}+c_{2}\right) x_{m}=x_{m} .
$$

Finally, the Hilbert projection theorem guarantees that for every $h \in H$, there exists a unique $y \in I$ such that $\|h-y\|$ is minimised as $y$ ranges over $I$. Since it is clear that $\theta \notin I$ we can take $h$ to be $\theta$. Then it follows that in the class $I$ of interpolants there exists one of smallest norm, and it is unique.

For an associated sampling theory in the finite dimensional case, see Yen [17, Theorem IV] in the classical setting; and Yau [16], Aronszajn [1, p. 346] and Saitoh [13, pp. 54, 193, 198] in the RK setting.

\section{ACKNOWLEDGEMENT}

The author takes pleasure in thanking Maurice Dodson for many helpful conversations, and two referees for their perceptive comments.

\section{REFERENCES}

[1] N. Aronszajn, Theory of reproducing kernels, Trans. Amer. Math. Soc. 68, 337-404, 1950.

[2] A. V. Balakrishnan, A note on the sampling principle for continuous signals, IRE Trans. Inform. Theory IT-3, 143-146, 1957.

[3] M.G. Beaty, M.M. Dodson, S.P. Eveson, and J.R. Higgins, On the approximate form of Kluvánek's theorem, J. Approx. Th. 160, 281-303, 2009.

[4] A. Berlinet, Reproducing kernels in probability and statistics, More progress in analysis. Proceedings of the 5th international ISAAC Congress, Catania, Italy, July 25-30, 2005. (H.G.W. Begehr et al., ed.), World Scientific, Hackensack, NJ,, 153-162, 2009.

[5] P.L. Butzer, P.J.S.G. Ferreira, J.R. Higgins, S. Saitoh, G. Schmeisser, and R.L. Stens, Interpolation and Sampling: E.T. Whittaker, K. Ogura and their followers, J. Fourier Anal. Appl. 17, 320-354, 2011.

[6] A.G. García and F.H. Szafraniec, A converse of the Kramer sampling theorem, Sampling Th. Signal and Image Proc. 1, no. 1, 53-61, 2002.

[7] J.R. Higgins, A sampling principle associated with Saitoh's fundamental theory of linear transformations, Analytic extension formulas and their applications (S. Saitoh et al., ed.), Kluwer Academic Publishers, Dordrecht, 73-85, 2001.

[8] J.R. Higgins, Sampling theory in Fourier and signal analysis: foundations, Clarendon Press, Oxford, 1996

[9] V.A. Kotel'nikov, On the transmission capacity of the "aether" and wire in electrocommunications, Material for the first All Union Conference on Questions of Communication (Moscow), Izd. Red. Upr. Svyazi rkKA, 1933, Russian.

[10] K. Ogura, On a certain transcendental integral function in the theory of interpolation, Tôhoku Math. J. 17, 64-72, 1920.

[11] _ On some central difference formulas of interpolation, Tôhoku Math. J. 17, 232$241,1920$.

[12] E. Parzen, Statistical inference on time series by RKHS methods, Proc. of 12th Biennial Seminar of the Canadian Mathematical Congress, Amer. Math. Soc., Providence, R.I. 1970.

[13] S. Saitoh, Integral transforms, reproducing kernels and their applications, Longman, Harlow, 1997. 
[14] J.M. Whittaker, The "Fourier" theory of the cardinal function, Proc. Edinburgh Math. Soc. 1, 169-176, 1929.

[15] - On the cardinal function of interpolation theory, Proc. Edinburgh Math. Soc. 1, 41-46, (1929).

[16] K. Yau, Application of reproducing kernel Hilbert spaces-bandlimited signal models, Information and Control 11, 429-444, 1967.

[17] J. L. Yen, On nonuniform sampling of bandwidth-limited signals, IRE Trans. circuit theory CT-3, 251-257, 1956.

[18] A. Zygmund, Trigonometrical series, Dover Publications, New York, 1955. 